2018

\title{
A New Lifetime Distribution For Series System: Model, Properties and Application
}

\author{
Adil Rashid \\ University of Kashmir, Srinagar, India, adilstat@gmail.com \\ Zahooor Ahmad \\ University of Kashmir, Srinagar, India, zahoorstat@gmail.com \\ T R.Jan \\ University of Kashmir, Srinagar, India, drtrjan@gmail.com
}

Follow this and additional works at: https://digitalcommons.wayne.edu/jmasm

Part of the Applied Statistics Commons, Social and Behavioral Sciences Commons, and the Statistical Theory Commons

\section{Recommended Citation}

Rashid, A., Ahmad, Z., \& Jan, T. R. (2018). A New Lifetime Distribution for Series System: Model, Properties and Application. Journal of Modern Applied Statistical Methods, 17(1), eP2535. doi: 10.22237/jmasm/1525133400

This Regular Article is brought to you for free and open access by the Open Access Journals at DigitalCommons@WayneState. It has been accepted for inclusion in Journal of Modern Applied Statistical Methods by an authorized editor of DigitalCommons@WayneState. 


\title{
A New Lifetime Distribution for Series System: Model, Properties and Application
}

\author{
Adil Rashid \\ University of Kashmir \\ Srinagar, India
}

\author{
Zahoor Ahmad \\ University of Kashmir \\ Srinagar, India
}

\author{
T. R. Jan \\ University of Kashmir \\ Srinagar, India
}

\begin{abstract}
A new lifetime distribution for modeling system lifetime in series setting is proposed that embodies most of the compound lifetime distribution. The reliability analysis of parent and of sub-models has also been discussed. Various mathematical properties that include moment generating function, moments, and order statistics have been obtained. The newlyproposed distribution has a flexible density function; more importantly its hazard rate function can take up different shapes such as bathtub, upside down bathtub, increasing, and decreasing shapes. The unknown parameters of the proposed generalized family have been estimated through MLE technique. The strength and usefulness of the proposed family was tested on a real life data set and it is quite clear from the statistical analysis that proposed family offers a better fit.
\end{abstract}

Keywords: Generalized Lindley distribution, power series distribution, compounding, hazard function, survival function

\section{Introduction}

The modeling of lifetime data has received prime attention from researchers for the last decade. Many continuous probability models such as exponential, gamma, and Weibull were frequently used in statistical literature to analyze the lifetime data, but these probability models cannot be used efficiently to model lifetime data that is bathtub shaped and have unimodal failure rates. To overcome this problem, researchers have focused their attention on compounding mechanisms which are an innovative way to construct suitable, flexible, and alternative models to fit the lifetime data of different types.

doi: 10.22237/jmasm/1525133400 | Accepted: June 5, 2017; Published: June 7, 2018.

Correspondence: Adil Rashid, adilstat@gmail.com 


\section{RASHID ET AL}

Consider a system with $N$ components, where $N$ is a discrete random variable with domain $N=1,2, \ldots$. The lifetime of the $i^{\text {th }}$ component is a continuous random variable, say $X_{i}$, that may follow any one of the lifetime distributions such as exponential, gamma, Weibull, Lindley, etc. The suitable discrete distributions for $N$ may be geometric, zero truncated Poisson, or power series distributions in general. The lifetime of such a system in series and parallel combination is defined and denoted by a non-negative random variable $Y=\min \left\{X_{i}\right\}_{i=1}^{N}$ or $Y=\max \left\{X_{i}\right\}_{i=1}^{N}$, respectively.

With this in mind, Adamidis and Loukas (1998) constructed a two parameter lifetime distribution by compounding the exponential distribution with the geometric distribution, called the Exponential Geometric (EG) distribution. Kus (2007) obtained a compound of the exponential distribution with that of Poisson and it was named the Exponential Poisson (EP) distribution. Tahmasbi and Rezaei (2008) obtained the Exponential Logarithmic (EL) distribution by using the same compounding mechanism. The extended EG distribution was considered by Adamidis, Dimitrakopoulou, and Loukas (2005).

Power series distributions contain several classical discrete distributions as special cases, therefore Chahkandi and Ganjali (2009) introduced a compound class of Exponential Power Series (EPS) distributions which contain several compound distributions as special cases. It is known that the Weibull distribution contains the exponential distribution as a special case; in view of this, Morais and Baretto-Souza (2011) replaced the exponential distribution with a Weibull distribution in the compounding mechanism of the EPS distribution and obtained a compound class of Weibull Power Series (WPS) distributions, which contains EPS distribution as a special case. Zakerzadeh and Mahmoudi (2012) obtained a two parameter lifetime distribution by compounding a Lindley distribution with a geometric distribution. Recently, a one parameter Lindley distribution has been used frequently to model lifetime data because it has been observed in several research papers that this distribution performs excellently well when it comes to fit the lifetime data. Adil and Jan (2016) introduced a new family of lifetime distributions which is obtained by compounding Lindley distribution with power series distribution. This new family of continuous lifetime distributions was called the Lindley Power Series (LPS) distribution.

The proposed LPS family of compound distributions contains several lifetime distributions as its special cases that are very flexible and able to accommodate different types of data sets since the probability density function and hazard rate can take on different forms such as increasing, decreasing, and upside down bathtub 


\section{A NEW LIFETIME DISTRIBUTION FOR SERIES SYSTEM}

shapes which have been shown through graphs for some selected values of parameters, and the potentiality of LPS family has been tested statistically by using it to model some real life data set.

There are many continuous distributions in statistics that can be used to model lifetime data; among them, the most popular are gamma, log normal, and Weibull distributions. The Weibull distribution has been used extensively by researchers to model lifetime data because of its closed form for survival function. However, all these lifetime model suffer from a major drawback, i.e., none of the them exhibit bathtub shapes for their hazard rate functions and hence cannot be used efficiently to model real life data that has bathtub shape for hazard rate function.

Nadarajah, Bakouch, and Tahmasbi (2011) proposed a new lifetime distribution called the generalized Lindley distribution that removes all of these mentioned drawbacks. It was shown that the generalized Lindley distribution has an attractive feature of allowing for monotonically decreasing, monotonically increasing, and bathtub shaped hazard rate functions, while not allowing for constant hazard rate functions. These important features of the generalized Lindley distribution attracted the attention of researchers Adil and Jan (2016), who replaced the Lindley distribution with a generalized Lindley distribution in the compounding mechanism of the LPS distribution.

\section{Construction of the Family}

Let $X_{1}, \ldots, X_{n}$ be an independent and identically distributed (iid) random variables following the generalized Lindley distribution due to Nadarajah et al. (2011), whose density function is given by

$$
\mathrm{g}(x ; \lambda)=\frac{\alpha \lambda^{2}}{\lambda+1}\left[1-\left(1+\frac{\lambda x}{\lambda+1}\right) \mathrm{e}^{-\lambda x}\right]^{\alpha-1} \mathrm{e}^{-\lambda x}(1+x), \quad x>0, \alpha, \lambda>0
$$

Here, the index $N$ is itself a discrete random variable following a zero truncated power series distribution with probability function given by

$$
\mathrm{P}(N=n)=\frac{a_{n} \theta^{n}}{\mathrm{C}(\theta)}, \quad n=1,2, \ldots
$$

where $a_{n}$ depends only on $n$, 


\section{RASHID ET AL}

Table 1. Useful quantities of some power series distributions

\begin{tabular}{rrrrrrr} 
Distribution & $\boldsymbol{a}_{\boldsymbol{n}}$ & $\mathbf{C}(\boldsymbol{\theta})$ & $\mathbf{C}^{\prime}(\boldsymbol{\theta})$ & $\mathbf{C}^{\prime \prime}(\boldsymbol{\theta})$ & $\mathbf{C}^{-1}(\boldsymbol{\theta})$ & $\boldsymbol{\theta}$ \\
\hline Poisson & $n !^{-1}$ & $\mathrm{e}^{\theta}-1$ & $\mathrm{e}^{\theta}$ & $\mathrm{e}^{\theta}$ & $\log (\theta+1)$ & $\theta \in(0, \infty)$ \\
Logarithmic & $n^{-1}$ & $-\log (1-\theta)$ & $(1-\theta)^{-1}$ & $(1-\theta)^{-2}$ & $1-\mathrm{e}^{-\theta}$ & $\theta \in(0,1)$ \\
Geometric & 1 & $\theta(1-\theta)^{-1}$ & $(1-\theta)^{-2}$ & $2(1-\theta)^{-3}$ & $\theta(\theta+1)^{-1}$ & $\theta \in(0,1)$ \\
Binomial & $\left(\begin{array}{c}m \\
n\end{array}\right)$ & $(\theta+1)^{m}-1$ & $m(\theta+1)^{m-1}$ & $\frac{m(m-1)}{(\theta+1)^{2-m}}$ & $(\theta-1)^{1 / m}-1$ & $\theta \in(0, \infty)$ \\
\hline
\end{tabular}

$$
\mathrm{C}(\theta)=\sum_{n=1}^{\infty} a_{n} \theta^{n}
$$

and $\theta$ is such that $\mathrm{C}(\theta)$ is finite. Table 1 shows useful quantities of some zero truncated power series distributions such as Poisson, logarithmic, geometric, and binomial (with $m$ being the number of replicas).

Let $X_{(1)}=\min \left\{X_{i}\right\}_{i=1}^{N}$. The conditional cumulative distribution function of $X_{(1)} \mid N=n$ is given by

$$
\begin{aligned}
G_{X_{(1)} \mid N=n} & =1-[\overline{\mathrm{G}}(x)]^{n} \\
G_{X_{(1)} \mid N=n} & =1-\left[1-\left[1-\left(1+\frac{\lambda x}{\lambda+1}\right) \mathrm{e}^{-\lambda x}\right]^{\alpha}\right]^{n}
\end{aligned}
$$

and

$$
\mathrm{p}\left(X_{(1)} \leq x, N=n\right)=\left\{1-\left[1-\left[1-\left(1+\frac{\lambda x}{\lambda+1}\right) \mathrm{e}^{-\lambda x}\right]^{\alpha}\right]^{n}\right\} \frac{a_{n} \theta^{n}}{\mathrm{C}(\theta)}, \quad x>0, n \geq 1
$$

The family of generalized Lindley power series distributions is defined by the marginal cumulative distribution function of $X_{(1)}$ : 


\section{A NEW LIFETIME DISTRIBUTION FOR SERIES SYSTEM}

$$
\begin{aligned}
\mathrm{F}(x) & =\sum_{n-1}^{\infty}\left\{1-\left[1-\left[1-\left(1+\frac{\lambda x}{\lambda+1}\right) \mathrm{e}^{-\lambda x}\right]^{\alpha}\right]^{n}\right\} \frac{a_{n} \theta^{n}}{\mathrm{C}(\theta)} \\
& =1-\frac{\mathrm{C}\left(\left[1-\left[1-\left(1+\frac{\lambda x}{\lambda+1}\right) \mathrm{e}^{-\lambda x}\right]^{\alpha}\right] \theta\right)}{\mathrm{C}(\theta)}
\end{aligned}
$$

\section{Density, Survival, and Hazard Rate Function}

The probability density function of a family of the Lindley power series (GLPS) distribution can be obtained by differentiating (2) both sides with respect to $x$.

$$
\begin{aligned}
\mathrm{f}(x)=\frac{\alpha \lambda^{2} \theta \mathrm{e}^{-\lambda x}}{(\lambda+1) \mathrm{C}(\theta)}[1 & \left.-\left(1+\frac{\lambda x}{\lambda+1}\right) \mathrm{e}^{-\lambda x}\right]^{\alpha-1}(1+x) \\
& \times \mathrm{C}^{\prime}\left(\left[1-\left[1-\left(1+\frac{\lambda x}{\lambda+1}\right) \mathrm{e}^{-\lambda x}\right]^{\alpha}\right] \theta\right)
\end{aligned}
$$

If $\alpha=1$ in (3), it reduces to a Lindley power series distribution. Alternatively, the probability density function of the GLPS distribution can also be obtained using the joint probability function of $X_{(1)}$ and $N$ :

$$
\begin{aligned}
\mathrm{g}_{X, N}(x, n)=n \frac{\alpha \lambda^{2}}{\lambda+1}(1+x) \mathrm{e}^{-\lambda x} \frac{a_{n} \theta^{n}}{\mathrm{C}(\theta)}\left[1-\left[1-\left(1+\frac{\lambda x}{\lambda+1}\right) \mathrm{e}^{-\lambda x}\right]^{\alpha}\right]^{n-1} & \times\left[1-\left(1+\frac{\lambda x}{\lambda+1}\right) \mathrm{e}^{-\lambda x}\right]^{\alpha+1}
\end{aligned}
$$

Therefore, density function of a family of the GLPS distribution is defined by the marginal density of $X_{(1)}$ : 


\section{RASHID ET AL}

$$
\begin{aligned}
\mathrm{f}(x)=\frac{\alpha \lambda^{2} \theta \mathrm{e}^{-\lambda x}}{(\lambda+1) \mathrm{C}(\theta)}[ & \left.1-\left(1+\frac{\lambda x}{\lambda+1}\right) \mathrm{e}^{-\lambda x}\right]^{\alpha-1}(1+x) \\
& \times \mathrm{C}^{\prime}\left(\left[1-\left[1-\left(1+\frac{\lambda x}{\lambda+1}\right) \mathrm{e}^{-\lambda x}\right]^{\alpha}\right] \theta\right)
\end{aligned}
$$

which is same as (3). The survival function of the GLPS distribution is given by

$$
\mathrm{S}(x)=\frac{\mathrm{C}\left(\left[1-\left[1-\left(1+\frac{\lambda x}{\lambda+1}\right) \mathrm{e}^{-\lambda x}\right]^{\alpha}\right] \theta\right)}{\mathrm{C}(\theta)}
$$

and the hazard function is

$$
\mathrm{h}(x)=\frac{\alpha \lambda^{2} \theta(1+x) \mathrm{e}^{-\lambda x}\left[1-\left(1+\frac{\lambda x}{\lambda+1}\right) \mathrm{e}^{-\lambda x}\right]^{\alpha-1} \mathrm{C}^{\prime}\left(\left[1-\left[1-\left(1+\frac{\lambda x}{\lambda+1}\right) \mathrm{e}^{-\lambda x}\right]^{\alpha}\right] \theta\right)}{(\lambda+1) \mathrm{C}\left(\left[1-\left[1-\left(1+\frac{\lambda x}{\lambda+1}\right) \mathrm{e}^{-\lambda x}\right]^{\alpha}\right] \theta\right)}
$$

Proposition 1. The generalized Lindley distribution is the limiting case of the proposed distribution when $\theta \rightarrow 0^{+}$.

Proof. From the cumulative distribution function of the GLPS distribution,

$$
\begin{aligned}
\lim _{\theta \rightarrow 0^{+}} \mathrm{F}(x) & =1-\lim _{\theta \rightarrow 0^{+}} 1-\frac{\mathrm{C}\left(\left[1-\left[1-\left(1+\frac{\lambda x}{\lambda+1}\right) \mathrm{e}^{-\lambda x}\right]^{\alpha}\right] \theta\right)}{\mathrm{C}(\theta)} \\
& =1-\lim _{\theta \rightarrow 0^{+}} \frac{\sum_{n=1}^{\infty} a_{n}\left(\left[1-\left[1-\left(1+\frac{\lambda x}{\lambda+1}\right) \mathrm{e}^{-\lambda x}\right]^{\alpha}\right] \theta\right)^{n}}{\sum_{n=1}^{\infty} a_{n} \theta^{n}}
\end{aligned}
$$




\section{A NEW LIFETIME DISTRIBUTION FOR SERIES SYSTEM}

as

$$
\mathrm{C}(\theta)=\sum_{n=1}^{\infty} a_{n} \theta^{n}
$$

and, using L'Hospital's rule, it follows that

$$
\begin{aligned}
\lim _{\theta \rightarrow 0^{+}} \mathrm{F}(x) & =1-\lim _{\theta \rightarrow 0^{+}} \frac{\sum_{n=1}^{\infty} a_{n} n \theta^{n-1}\left[1-\left[1-\left(1+\frac{\lambda x}{\lambda+1}\right) \mathrm{e}^{-\lambda x}\right]^{\alpha}\right]^{n}}{\sum_{n=1}^{\infty} a_{n} n \theta^{n-1}} \\
& =\left[1-\left(1+\frac{\lambda x}{\lambda+1}\right) \mathrm{e}^{-\lambda x}\right]^{\alpha}
\end{aligned}
$$

which is the distribution function of the generalized Lindley distribution.

Proposition 2. The densities of proposed distribution can be expressed as an infinite linear combination of densities of $1^{\text {st }}$ order statistics of the generalized Lindley distribution

$$
\mathrm{f}(x)=\sum_{n=1}^{\infty} \mathrm{P}(N=n) \mathrm{g}_{1}(x, n)
$$

where

$$
\begin{aligned}
\mathrm{g}_{1}(x, n)=n \frac{\alpha \lambda^{2}}{\lambda+1}(1+x) \mathrm{e}^{-\lambda x}[1-(1 & \left.\left.+\frac{\lambda x}{\lambda+1}\right) \mathrm{e}^{-\lambda x}\right]^{\alpha-1} \\
& \left.\times\left(\left[1-\left[1-\left(1+\frac{\lambda x}{\lambda+1}\right) \mathrm{e}^{-\lambda x}\right]\right]^{\alpha}\right]\right)^{n-1}
\end{aligned}
$$

Proof. It is known that

$$
\mathrm{C}^{\prime}(\theta)=\sum_{n=1}^{\infty} n a_{n} \theta^{n-1}
$$




\section{RASHID ET AL}

Therefore,

$$
\begin{aligned}
\mathrm{f}(x)= & \frac{\alpha \lambda^{2}}{\lambda+1}(1+x) \mathrm{e}^{-\lambda x}\left[1-\left(1+\frac{\lambda x}{\lambda+1}\right) \mathrm{e}^{-\lambda x}\right]^{\alpha-1} \\
& \times \sum_{n=1}^{\infty} \frac{a_{n} \theta^{n}}{\mathrm{C}(\theta)} n\left(\left[1-\left[1-\left(1+\frac{\lambda x}{\lambda+1}\right) \mathrm{e}^{-\lambda x}\right]^{\alpha}\right]\right)^{n-1} \\
= & \sum_{n=1}^{\infty} \mathrm{P}(N=n) \mathrm{g}_{1}(x, n)
\end{aligned}
$$

where $g_{1}(\mathrm{x}, \mathrm{n})$, defined as in the statement of the proposition, is the pdf of $X_{(1)}=\min \left(X_{1}, X_{2}, \ldots, X_{n}\right)$. Therefore the densities of the proposed distribution can be expressed as an infinite linear combination of the $1^{\text {st }}$ order statistics of the generalized Lindley distribution.

\section{Moment Generating Function}

The moment generating function (mgf) of the proposed distribution can be obtained from (5):

$$
\mathrm{M}_{X}(t)=\sum_{n=1}^{\infty} \mathrm{P}(N=n) \mathrm{M}_{X_{(1)}}(t)
$$

where $\mathrm{M}_{X_{(1)}}(t)$ is the moment generating function of $1^{\text {st }}$ order statistics of the generalized Lindley distribution:

$$
\begin{aligned}
\mathrm{M}_{X_{(1)}}(t)=\int_{0}^{\infty} \mathrm{e}^{t x} n \frac{\alpha \lambda^{2}}{\lambda+1}(1+x) & \mathrm{e}^{-\lambda x}\left[1-\left(1+\frac{\lambda x}{\lambda+1}\right) \mathrm{e}^{-\lambda x}\right]^{\alpha-1} \\
\times & \left(\left[1-\left[1-\left(1+\frac{\lambda x}{\lambda+1}\right) \mathrm{e}^{-\lambda x}\right]^{\alpha}\right]\right)^{n-1} d x
\end{aligned}
$$




\section{A NEW LIFETIME DISTRIBUTION FOR SERIES SYSTEM}

$$
\begin{aligned}
\mathrm{M}_{X_{(1)}}(t)= & n \frac{\alpha \lambda^{2}}{\lambda+1} \sum_{j=0}^{n-1}\left(\begin{array}{c}
n-1 \\
j
\end{array}\right)(-1)^{j} \int_{0}^{\infty} \mathrm{e}^{t x-\lambda x}(1+x) \\
& \times\left[1-\left(1+\frac{\lambda x}{\lambda+1}\right) \mathrm{e}^{-\lambda x}\right]^{\alpha j}
\end{aligned}
$$

Hence

$$
\begin{aligned}
\mathrm{M}_{X}(t)=\frac{\alpha \lambda^{2}}{\lambda+1} \sum_{n=1}^{\infty} \frac{a_{n} \theta^{n}}{\mathrm{C}(\theta)} n \sum_{j=0}^{n-1} \sum_{k=0}^{\infty} \sum_{i=0}^{k}\left(\begin{array}{c}
n-1 \\
j
\end{array}\right)\left(\begin{array}{c}
\alpha j+\alpha-1 \\
k
\end{array}\right)\left(\begin{array}{l}
k \\
i
\end{array}\right)\left(\frac{\lambda}{\lambda+1}\right)^{k-i}(-1)^{j+k} \\
\times\left(\frac{\Gamma(k-i+1)(\lambda k+\lambda-t)^{k-i+1}+\Gamma(k-i+2)}{(\lambda k+\lambda-t)^{k-i+2}}\right)
\end{aligned}
$$

As

$$
\begin{aligned}
\mathrm{E}\left(X_{(1)}\right)=\sum_{j=0}^{n-1} \sum_{k=0}^{\infty} \sum_{i=0}^{k}\left(\begin{array}{c}
n-1 \\
j
\end{array}\right)\left(\begin{array}{c}
\alpha j+\alpha-1 \\
k
\end{array}\right)\left(\begin{array}{l}
k \\
i
\end{array}\right)(-1)^{j+k} \frac{n \alpha \lambda^{k-i+2}}{(\lambda+1)^{k-i+1}} \\
\times\left[\frac{\Gamma(m+k-i+1)}{(\lambda k+\lambda)^{m+k-i+1}}+\frac{\Gamma(m+k-i+2)}{(\lambda k+\lambda)^{m+k-i+2}}\right]
\end{aligned}
$$




\section{RASHID ET AL}

the $m^{\text {th }}$ moment of proposed distribution about origin is

$$
\begin{aligned}
\mathrm{E}\left(X^{m}\right)= & \sum_{n=1}^{\infty} \mathrm{P}(N=n) \int_{0}^{\infty} x^{m} \mathrm{~g}_{1}(x) d x \\
= & \sum_{n=1}^{\infty} \mathrm{P}(N=n) \mathrm{E}\left(X_{(1)}\right) \\
= & \sum_{n=1}^{\infty} \sum_{j=0}^{n-1} \sum_{k=0}^{\infty} \sum_{i=0}^{k} \frac{a_{n} \theta^{n}}{\mathrm{C}(\theta)}\left(\begin{array}{c}
n-1 \\
j
\end{array}\right)\left(\begin{array}{c}
\alpha j+\alpha-1 \\
k
\end{array}\right)\left(\begin{array}{l}
k \\
i
\end{array}\right)(-1)^{j+k} \frac{n \alpha \lambda^{k-i+2}}{(\lambda+1)^{k-i+1}} \\
& \times\left[\frac{\Gamma(m+k-i+1)}{(\lambda k+\lambda)^{m+k-i+1}}+\frac{\Gamma(m+k-i+2)}{(\lambda k+\lambda)^{m+k-i+2}}\right]
\end{aligned}
$$

\section{Order Statistics and Their Moments}

Let $X_{1}, X_{2}, \ldots, X_{n}$ be a random sample from the GLPS distribution, and let $X_{1: n}<X_{2: n}<\ldots<X_{n: n}$ denote the corresponding order statistics. The pdf of the $i^{\text {th }}$ order statistic $X_{i: n}$ is given by

$$
\begin{aligned}
& \mathrm{f}_{i: n}(x)=\frac{n ! \mathrm{f}(x)}{(n-i) !(i-1) !}\left[1-\frac{\mathrm{C}\left(\left[1-\left[1-\left(1+\frac{\lambda x}{\lambda+1}\right) \mathrm{e}^{-\lambda x}\right]^{\alpha}\right]\right.}{\mathrm{C}(\theta)}\right]^{i-1} \\
& \times {\left[\frac{\mathrm{C}\left(\left[1-\left[1-\left(1+\frac{\lambda x}{\lambda+1}\right) \mathrm{e}^{-\lambda x}\right]^{\alpha}\right] \theta\right)}{\mathrm{C}(\theta)}\right]^{n-i} }
\end{aligned}
$$

From

$$
\mathrm{f}(x)[\mathrm{F}(x)]^{k+i-1}=\left(\frac{1}{k+i}\right) \frac{d}{d x}[\mathrm{~F}(x)]^{k+i}
$$




\section{A NEW LIFETIME DISTRIBUTION FOR SERIES SYSTEM}

the associated CDF is

$$
\begin{aligned}
\mathrm{F}_{i: n}(x)=\frac{n !}{(n-i) !(i-1) !} \sum_{k=0}^{n-i} \frac{\left(\begin{array}{c}
n-i \\
k
\end{array}\right)(-1)^{k}}{(k+i)} & \\
& {\left.\left.\left[1-\frac{\mathrm{C}\left(\left[1-\left[1-\left(1+\frac{\lambda x}{\lambda+1}\right) \mathrm{e}^{-\lambda x}\right]\right.\right.}{\mathrm{C}(\theta)}\right] \theta\right)\right]^{\alpha+i} }
\end{aligned}
$$

Alternatively equation (8) can be written as

$$
\begin{aligned}
\mathrm{F}_{i: n}(x)=1-\frac{n !}{(n-i) !(i-1) !} \sum_{k=0}^{i-1} & \frac{\left(\begin{array}{c}
i-1 \\
k
\end{array}\right)(-1)^{k}}{(k+i)} \\
\times & {\left[\frac{\mathrm{C}\left(\left[1-\left[1-\left(1+\frac{\lambda x}{\lambda+1}\right) \mathrm{e}^{-\lambda x}\right]^{\alpha}\right] \theta\right)}{\mathrm{C}(\theta)}\right]^{k+n-i+1} }
\end{aligned}
$$

The expression for the $r^{\text {th }}$ moment of $i^{\text {th }}$ order statistic $X_{i: n}$ with CDF (8) can be obtained, using a well-known result due to Barakat and Abdelkadir (2004), as

$$
\mathrm{E}\left(X_{i: n}^{r}\right)=r \sum_{k=n-i+1}^{n} \frac{(-1)^{k-n+i-1}}{\mathrm{C}(\theta)^{k}}\left(\begin{array}{l}
k-1 \\
n-i
\end{array}\right)\left(\begin{array}{l}
n \\
k
\end{array}\right) \int_{0}^{\infty} x^{r-1} \mathrm{C}\left(\left[1-\left[1-\left(1+\frac{\lambda x}{\lambda+1}\right) \mathrm{e}^{-\lambda x}\right]^{\alpha}\right] \theta\right)^{k} d x
$$

where $r=1,2,3, \ldots$ and $i=1,2, \ldots, n$. 


\section{RASHID ET AL}

\section{Parameter Estimation}

Let $X_{1}, \ldots, X_{N}$ be a random sample with observed values $x_{1}, \ldots, x_{n}$ from a GLPS distribution, and let $\boldsymbol{\Theta}=(\alpha, \lambda, \theta)^{\mathrm{T}}$ be the unknown parameter vector. The loglikelihood function is given by

$$
\begin{aligned}
l_{n}= & l_{n}(x, \boldsymbol{\Theta}) \\
= & n \log \alpha+2 n \log \lambda+n \log \theta-n \log (\lambda+1)+\sum_{i=1}^{n} \log \left(1+x_{i}\right) \\
& -n \log \mathrm{C}(\theta)-\lambda \sum_{i=1}^{n} x_{i}+(\alpha-1) \sum_{i=1}^{n} \log \left[1-\left(1+\frac{\lambda x_{i}}{\lambda+1}\right) \mathrm{e}^{-\lambda x_{i}}\right] \\
& +\sum_{i=1}^{n} \log \mathrm{C}^{\prime}\left(\left[1-\left[1-\left(1+\frac{\lambda x_{i}}{\lambda+1}\right) \mathrm{e}^{-\lambda x_{i}}\right]^{\alpha}\right] \theta\right)
\end{aligned}
$$

The corresponding score functions are

$$
\begin{gathered}
\frac{\partial l_{n}}{\partial \theta}=\frac{n}{\theta}-\frac{n \mathrm{C}^{\prime}(\theta)}{\mathrm{C}(\theta)}+\sum_{i=1}^{n} \frac{\mathrm{C}^{\prime \prime}\left(\theta \mathrm{e}^{-\lambda x}\left(1+\frac{\lambda x}{\lambda+1}\right)\right)}{\mathrm{C}^{\prime}\left(\theta \mathrm{e}^{-\lambda x}\left(1+\frac{\lambda x}{\lambda+1}\right)\right)}\left[1-\left(1-\left(1+\frac{\lambda x}{\lambda+1}\right) \mathrm{e}^{-\lambda x}\right)^{\alpha}\right] \\
\left.\left.\frac{\partial l_{n}}{\partial \alpha}=\frac{n}{\alpha}-\alpha \theta \sum_{i=1}^{n} \frac{\mathrm{C}^{\prime \prime}\left(\left[1-\left[1-\left(1+\frac{\lambda x}{\lambda+1}\right) \mathrm{e}^{-\lambda x}\right]\right.\right.}{\mathrm{C}^{\prime}\left(\left[1-\left[1-\left(1+\frac{\lambda x}{\lambda+1}\right) \mathrm{e}^{-\lambda x}\right]\right.\right.}\right] \theta\right)\left(1-\left(1+\frac{\lambda x}{\lambda+1}\right) \mathrm{e}^{-\lambda x}\right)^{\alpha} \\
\times \log \left(1-\left(1+\frac{\lambda x}{\lambda+1}\right) \mathrm{e}^{-\lambda x}\right)+\sum_{i=1}^{n} \log \left(1-\left(1+\frac{\lambda x}{\lambda+1}\right) \mathrm{e}^{-\lambda x}\right)
\end{gathered}
$$




\section{A NEW LIFETIME DISTRIBUTION FOR SERIES SYSTEM}

$$
\begin{aligned}
\frac{\partial l_{n}}{\partial \lambda}=\frac{2 n}{\lambda}-\sum_{i=1}^{n} x_{i}-\frac{n}{\lambda+1}+\frac{(\alpha-1)}{(\lambda+1)^{2}} \sum_{i=1}^{n}\left(\frac{\left(\lambda(\lambda+1)^{2}+\lambda^{2} x(\lambda+1)-x\right) \mathrm{e}^{-\lambda x}}{1-\left(1+\frac{\lambda x}{\lambda+1}\right) \mathrm{e}^{-\lambda x}}\right) \\
+\frac{\alpha \theta}{(\lambda+1)^{2}} \mathrm{e}^{-\lambda x}(x-\lambda(\lambda+1)(\lambda x+\lambda+1)) \\
\quad \times \sum_{i=1}^{n} \frac{\mathrm{C}^{\prime \prime}\left(\left[1-\left[1-\left(1+\frac{\lambda x}{\lambda+1}\right) \mathrm{e}^{-\lambda x}\right]^{\alpha}\right] \theta\right)}{\mathrm{C}^{\prime}\left(\left[1-\left[1-\left(1+\frac{\lambda x}{\lambda+1}\right) \mathrm{e}^{-\lambda x}\right]^{\alpha}\right] \theta\right)}\left(1-\left(1+\frac{\lambda x}{\lambda+1}\right) \mathrm{e}^{-\lambda x}\right)^{\alpha-1}
\end{aligned}
$$

The maximum likelihood estimate of $\boldsymbol{\Theta}$, say $\hat{\boldsymbol{\Theta}}$, is obtained by solving the nonlinear system of equations

$$
\boldsymbol{\Theta}=\left(\frac{\partial l_{n}}{\partial \alpha}, \frac{\partial l_{n}}{\partial \lambda}, \frac{\partial l_{n}}{\partial \theta}\right)^{\mathrm{T}}=0
$$

The solution of this non-linear system of equation can be found numerically by using R.

\section{Some Special Sub-Models}

\section{Generalized Lindley Poisson Distribution}

The Poisson distribution is a special case of the power series distribution for $\mathrm{C}(\theta)=\mathrm{e}^{\theta}-1$; therefore the cdf of a compound of the generalized Lindley Poisson (GLP) distribution is obtained by using the same arguments in (2):

$$
\mathrm{F}(x)=1-\frac{\mathrm{e}^{\left.\left(1-\left[1-\left(1+\frac{\lambda x}{\lambda+1}\right) \mathrm{e}^{-\lambda x}\right]\right]^{\alpha}\right)}-1}{\mathrm{e}^{\theta}-1}, \quad x>0
$$

The associated pdf, hazard, and survival functions are given by, respectively, 


\section{RASHID ET AL}

$$
\begin{aligned}
& \mathrm{f}(x)=\frac{\alpha \lambda^{2} \theta \mathrm{e}^{-\lambda x}}{(\lambda+1) \mathrm{e}^{\theta-1}}\left[1-\left(1+\frac{\lambda x}{\lambda+1}\right) \mathrm{e}^{-\lambda x}\right]^{\alpha-1}(1+x) \mathrm{e}^{\left(1-\left[1-\left(1+\frac{\lambda x}{\lambda+1}\right) \mathrm{e}^{-\lambda x}\right]^{\alpha}\right) \theta} \\
& \mathrm{h}(x)=\frac{\alpha \lambda^{2} \theta \mathrm{e}^{-\lambda x}\left[1-\left(1+\frac{\lambda x}{\lambda+1}\right) \mathrm{e}^{-\lambda x}\right]^{\alpha-1}(1+x) \mathrm{e}^{\left(1-\left[1-\left(1+\frac{\lambda x}{\lambda+1}\right) e^{-\lambda x}\right]^{\alpha}\right) \theta}}{(\lambda+1)\left(\mathrm{e}^{\left(1-\left[1-\left(1+\frac{\lambda x}{\lambda+1}\right) \mathrm{e}^{-\lambda x}\right]^{\alpha}\right) \theta}-1\right)} \\
& \mathrm{S}(x)=\frac{\mathrm{e}^{\left(1-\left[1-\left(1+\frac{\lambda x}{\lambda+1}\right) \mathrm{e}^{-\lambda x}\right]^{\alpha}\right) \theta}-1}{\mathrm{e}^{\theta}-1}
\end{aligned}
$$
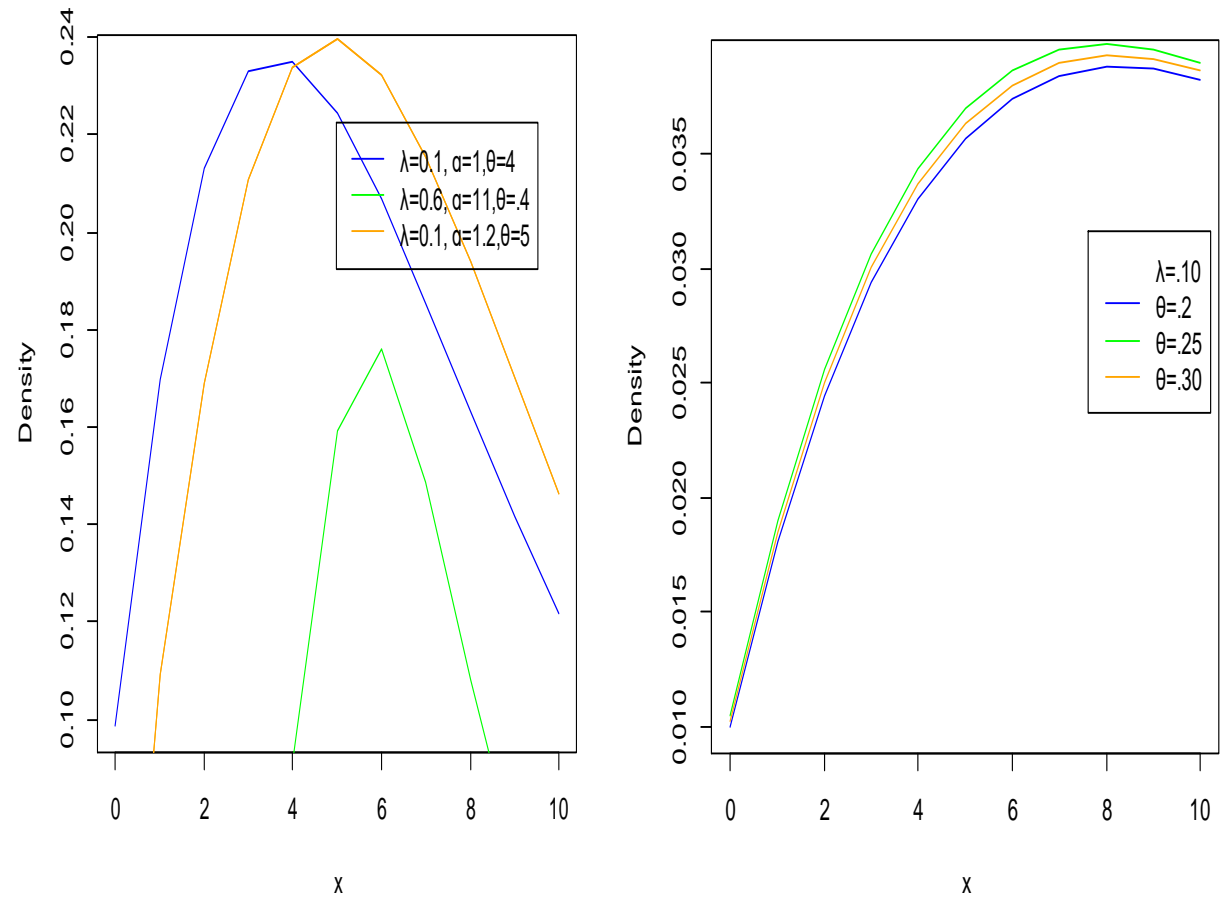

Figure 1. The flexibility of density of the GLP and LP distributions for some selected values of parameters 


\section{A NEW LIFETIME DISTRIBUTION FOR SERIES SYSTEM}
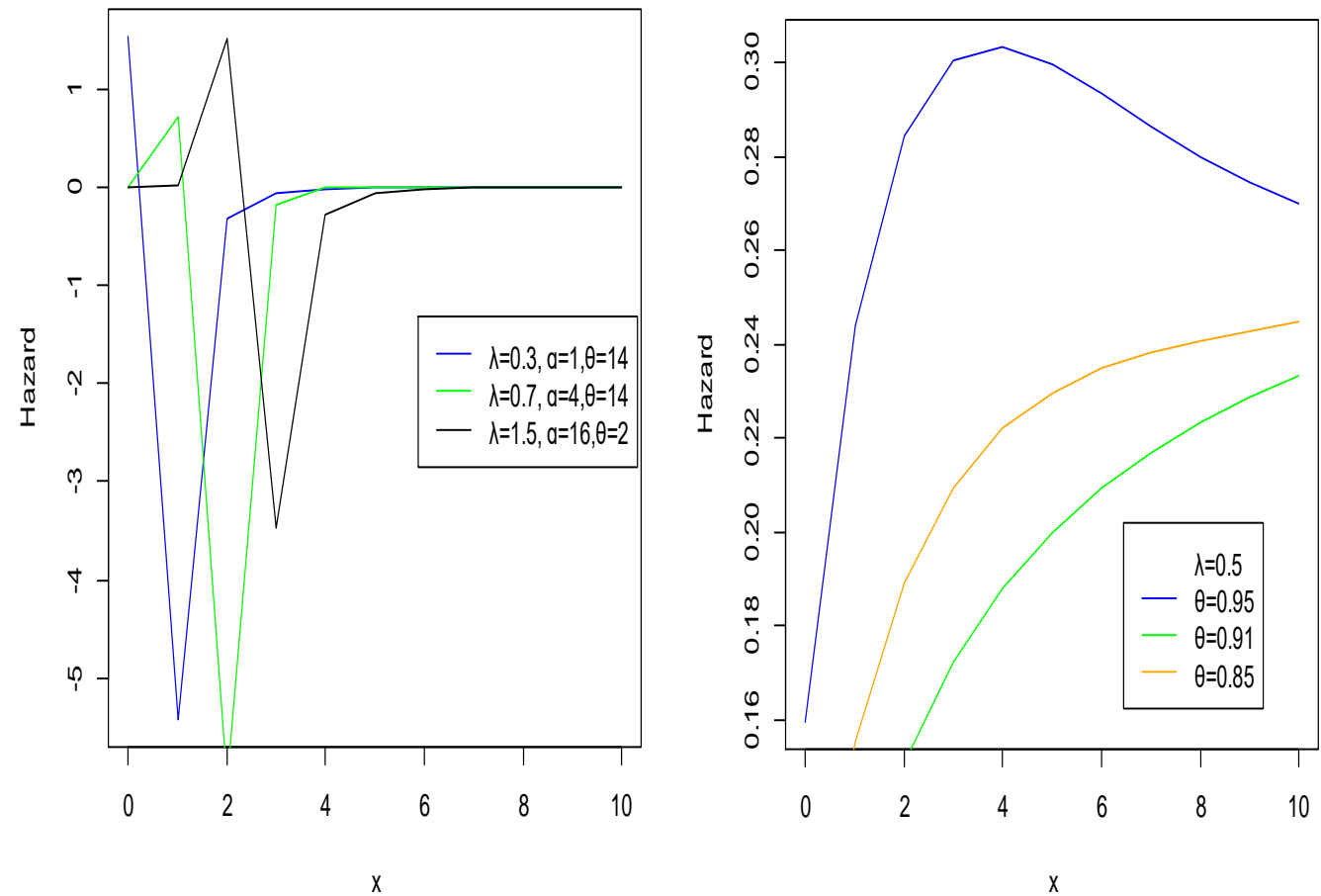

Figure 2. The flexibility of the hazard rate functions of the GLP and LP distributions for some selected values of parameters

for $x, \alpha, \lambda>0$ and $0<\theta<\infty$, respectively. The GLP distribution is very flexible in terms of its density and hazard rate function, which is also corroborated by Figure 1 and Figure 2. The expression for the $m^{\text {th }}$ moment of a random variable following the generalized Lindley Poisson distribution becomes, by taking $a_{n}=n !^{-1}$ and $\mathrm{C}(\theta)=\mathrm{e}^{\theta}-1$ in $(6)$,

$$
\begin{aligned}
\mathrm{E}\left(X^{m}\right)=\left(\mathrm{e}^{\theta}-1\right)^{-1} \sum_{n=1}^{\infty} \sum_{j=0}^{n-1} \sum_{k=0}^{\infty} \sum_{i=0}^{k} \frac{\theta^{n}}{n !}\left(\begin{array}{c}
n-1 \\
j
\end{array}\right)\left(\begin{array}{c}
\alpha j+\alpha-1 \\
k
\end{array}\right)\left(\begin{array}{l}
k \\
i
\end{array}\right)(-1)^{j+k} \frac{n \alpha \lambda^{k-i+2}}{(\lambda+1)^{k-i+1}} \\
\times\left[\frac{\Gamma(m+k-i+1)}{(\lambda k+\lambda)^{m+k-i+1}}+\frac{\Gamma(m+k-i+2)}{(\lambda k+\lambda)^{m+k-i+2}}\right]
\end{aligned}
$$

for $\alpha, \lambda>0$ and $0<\theta<\infty$. 


\section{RASHID ET AL}
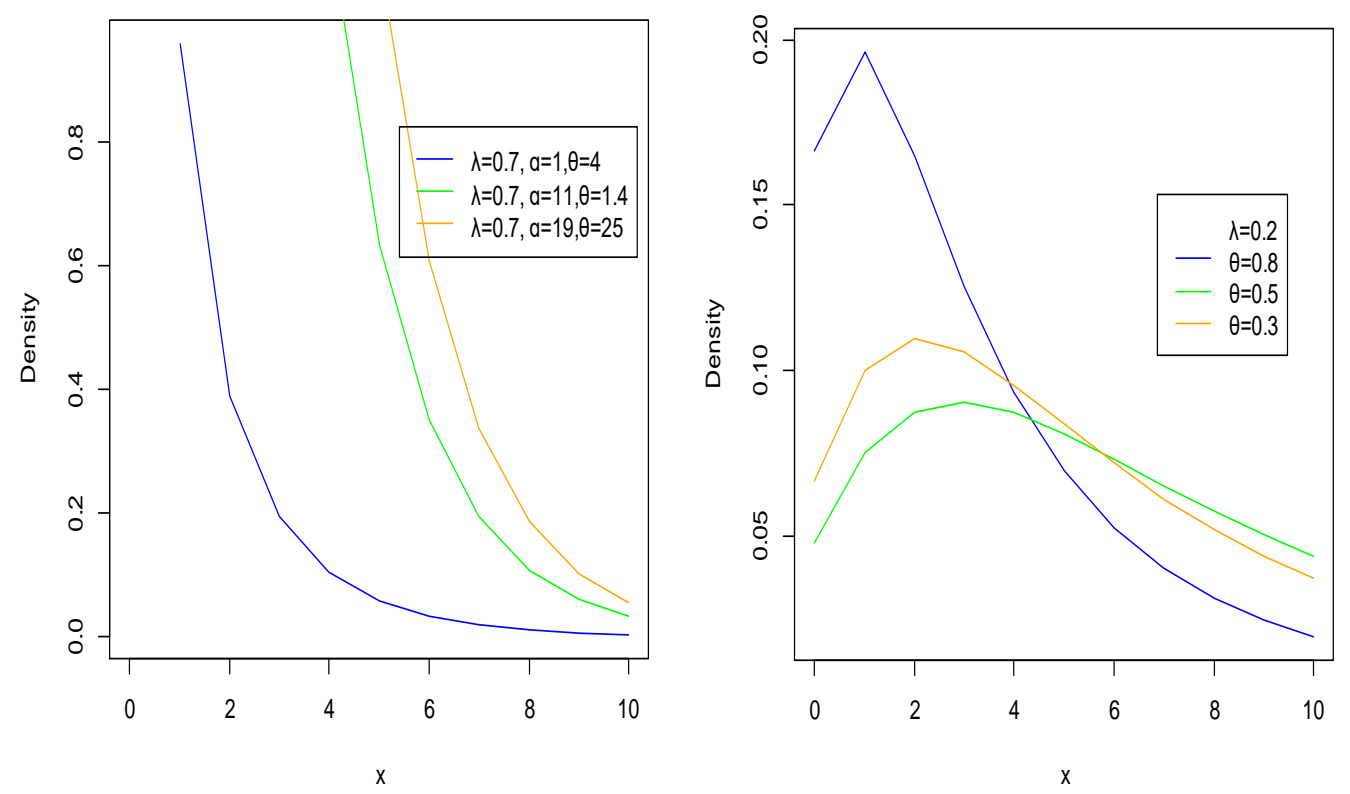

Figure 3. The flexibility of the density function of the GLL and LL distributions for some selected values of parameters

\section{Generalized Lindley Logarithmic Distribution}

The logarithmic distribution is a special case of the PSD when $C(\theta)=-\log (1-\theta)$, and a compound of the generalized Lindley logarithmic (GLL) distribution follows from (2) from the above $\mathrm{C}(\theta)$ :

$$
\mathrm{F}(x)=1-\frac{\log \left(\left(1-\left[1-\left(1+\frac{\lambda x}{\lambda+1}\right) \mathrm{e}^{-\lambda x}\right]^{\alpha}\right) \theta\right)}{\log (1-\theta)}
$$

The associated pdf, hazard rate, and survival functions are 


\section{A NEW LIFETIME DISTRIBUTION FOR SERIES SYSTEM}

$$
\begin{aligned}
& \mathrm{f}(x)=\frac{\alpha \lambda^{2} \theta \mathrm{e}^{-\lambda x}\left[1-\left(1+\frac{\lambda x}{\lambda+1}\right) \mathrm{e}^{-\lambda x}\right]^{\alpha-1}(1+x)}{(\lambda+1)\left(\left[1-\left[1-\left(1+\frac{\lambda x}{\lambda+1}\right) \mathrm{e}^{-\lambda x}\right]^{\alpha}\right] \theta-1\right) \log (1-\theta)} \\
& \mathrm{h}(x)=\frac{\left.\alpha \lambda^{2} \theta \mathrm{e}^{-\lambda x}\left[1-\left(1+\frac{\lambda x}{\lambda+1}\right) \mathrm{e}^{-\lambda x}\right]^{\alpha-1}(1+x)\left(\left[1-\left[1-\left(1+\frac{\lambda x}{\lambda+1}\right) \mathrm{e}^{-\lambda x}\right]\right]^{\alpha}\right] \theta-1\right)^{-1}}{(\lambda+1) \log \left(1-\left[1-\left[1-\left(1+\frac{\lambda x}{\lambda+1}\right) \mathrm{e}^{-\lambda x}\right]^{\alpha}\right] \theta\right)} \\
& \mathrm{S}(x)=\frac{\log \left(1-\left[1-\left[1-\left(1+\frac{\lambda x}{\lambda+1}\right) \mathrm{e}^{-\lambda x}\right]^{\alpha}\right] \theta\right)}{\log (1-\theta)}
\end{aligned}
$$

The density and hazard rate functions of the GLL distribution take different shapes for different values of parameters, which is evident from Figures 3 and 4. The expression for the $m^{\text {th }}$ moment of a random variable following a generalized Lindley logarithmic distribution becomes, by taking $a_{n}=n^{-1}$ and $\mathrm{C}(\theta)=-\log (1-\theta)$ in (6),

$$
\begin{aligned}
\mathrm{E}\left(X^{m}\right)=\frac{1}{\log (1-\theta)} \sum_{n=1}^{-1} \sum_{j=0}^{n-1} \sum_{k=0}^{\infty} \sum_{i=0}^{k} \theta^{n}\left(\begin{array}{c}
n-1 \\
j
\end{array}\right)\left(\begin{array}{c}
\alpha j+\alpha-1 \\
k
\end{array}\right)\left(\begin{array}{l}
k \\
i
\end{array}\right)(-1)^{j+k} \frac{\alpha \lambda^{k-i+2}}{(\lambda+1)^{k-i+1}} \\
\times\left[\frac{\Gamma(m+k-i+1)}{(\lambda k+\lambda)^{m+k-i+1}}+\frac{\Gamma(m+k-i+2)}{(\lambda k+\lambda)^{m+k-i+2}}\right]
\end{aligned}
$$

for $\alpha, \lambda>0$ and $0<\theta<1$. 


\section{RASHID ET AL}
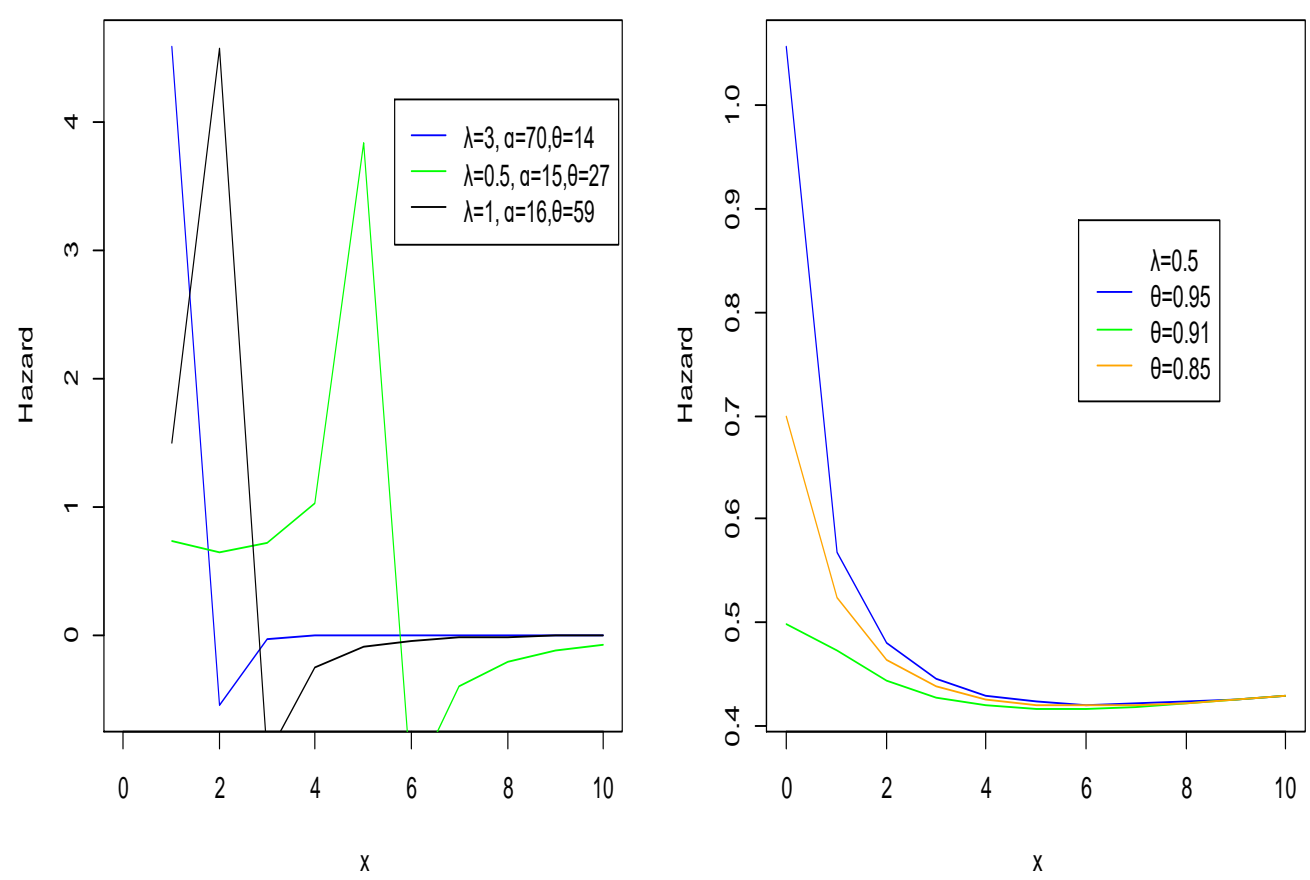

Figure 4. The flexibility of the hazard rate function of the GLL and LL distributions for some selected values of parameters

\section{Generalized Lindley Geometric Distribution}

The geometric distribution is a particular case of the PSD when $\mathrm{C}(\theta)=\theta(1-\theta)^{-1}$, and a compound of the generalized Lindley geometric distribution followed from (2):

$$
\mathrm{F}(x)=1-(1-\theta) \frac{\left(1-\left[1-\left(1+\frac{\lambda x}{\lambda+1}\right) \mathrm{e}^{-\lambda x}\right]^{\alpha}\right)}{\left(1-\left[1-\left[1-\left(1+\frac{\lambda x}{\lambda+1}\right) \mathrm{e}^{-\lambda x}\right]^{\alpha}\right] \theta\right)}, \quad x>0
$$

The associated pdf, hazard rate, and survival functions are 


\section{A NEW LIFETIME DISTRIBUTION FOR SERIES SYSTEM}
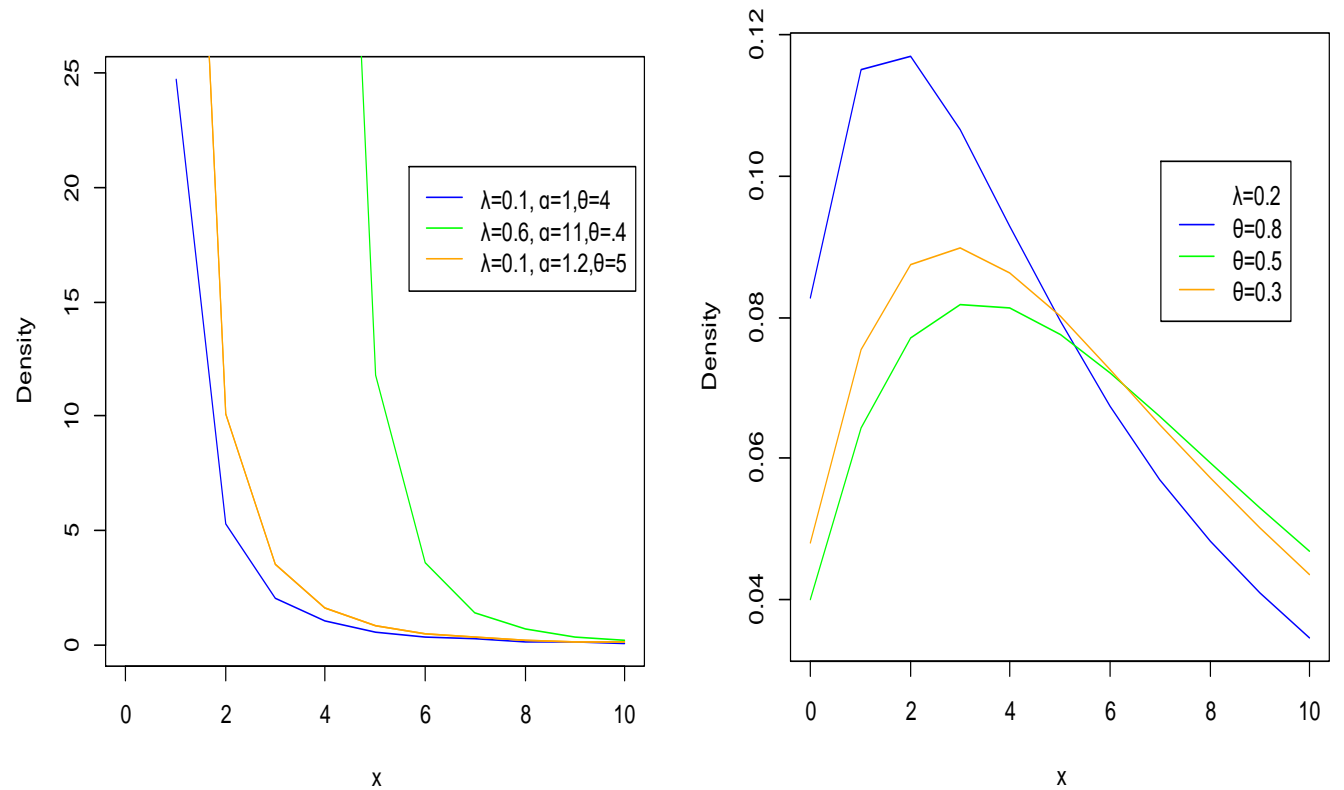

Figure 5. The flexibility of the density of the GLG and LG distributions for some selected values of parameters

$$
\begin{aligned}
& \mathrm{f}(x)=\frac{\alpha \lambda^{2}(1-\theta)(1+x) \mathrm{e}^{-\lambda x}\left[1-\left(1+\frac{\lambda x}{\lambda+1}\right) \mathrm{e}^{-\lambda x}\right]^{\alpha-1}}{(\lambda+1)\left(\left[1-\left[1-\left(1+\frac{\lambda x}{\lambda+1}\right) \mathrm{e}^{-\lambda x}\right]^{\alpha}\right] \theta\right)^{2}} \\
& \left.\mathrm{~h}(x)=\frac{\alpha \lambda^{2}(1+x) \mathrm{e}^{-\lambda x}\left[1-\left(1+\frac{\lambda x}{\lambda+1}\right) \mathrm{e}^{-\lambda x}\right]^{\alpha-1}}{(\lambda+1)\left(1-\left[1-\left(1+\frac{\lambda x}{\lambda+1}\right) \mathrm{e}^{-\lambda x}\right]^{\alpha}\right)\left(1-\left[1-\left[1-\left(1+\frac{\lambda x}{\lambda+1}\right) \mathrm{e}^{-\lambda x}\right]^{\alpha}\right]\right.}\right) \\
& \mathrm{S}(x)=\frac{(1-\theta)\left(1-\left[1-\left(1+\frac{\lambda x}{\lambda+1}\right) \mathrm{e}^{-\lambda x}\right]^{\alpha}\right)}{\left(1-\left[1-\left[1-\left(1+\frac{\lambda x}{\lambda+1}\right) \mathrm{e}^{-\lambda x}\right]^{\alpha}\right] \theta\right)}
\end{aligned}
$$




\section{RASHID ET AL}
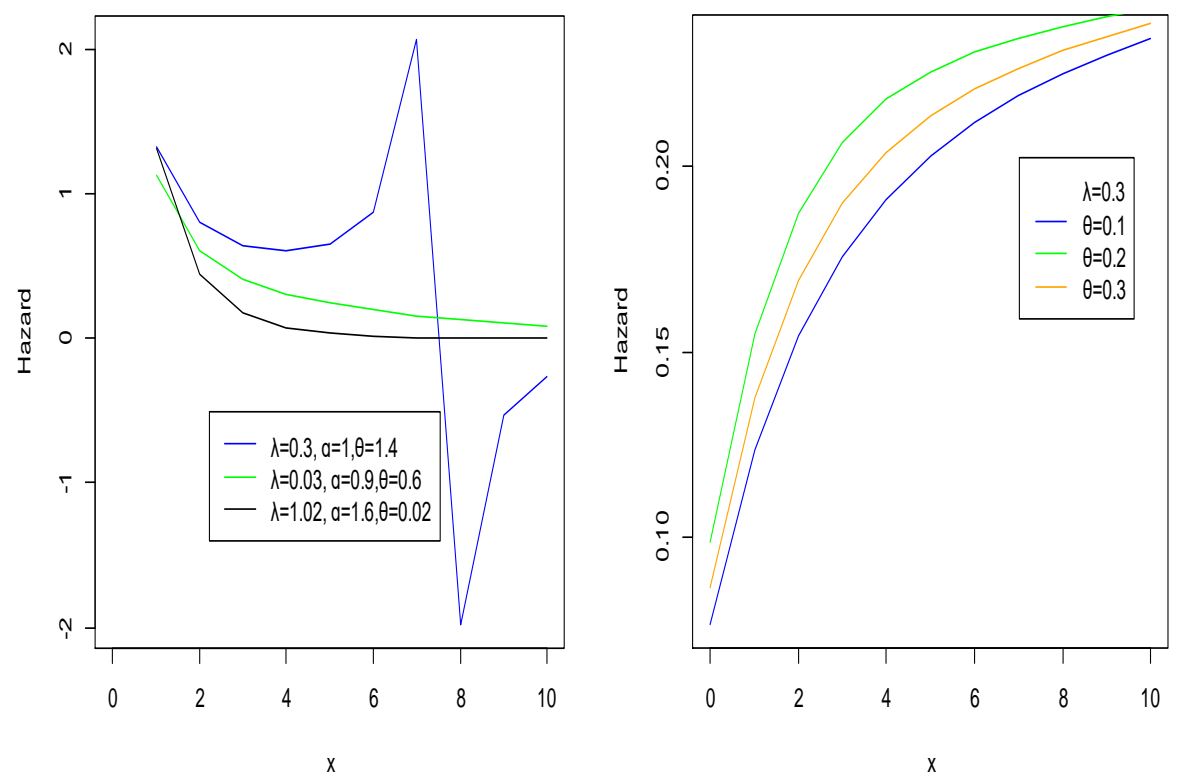

Figure 6. The flexibility of the hazard rate function of the GLG and LG distributions for some selected values of parameters

for $x, \alpha, \lambda>0$ and $0<\theta<1$, respectively. Figure 5 and Figure 6 show that the density and hazard rate functions of the GLG distribution take different shapes for different values of parameters. The expression for the $m^{\text {th }}$ moment of a random variable following a generalized Lindley geometric (GLG) distribution becomes, by taking $a_{n}=1$ and $\mathrm{C}(\theta)=\theta(1-\theta)^{-1}$ in (6),

$$
\begin{aligned}
\mathrm{E}\left(X^{m}\right)=\sum_{n=1}^{\infty} \sum_{j=0}^{n-1} \sum_{k=0}^{\infty} \sum_{i=0}^{k} \frac{\theta^{n-1}}{(1-\theta)}\left(\begin{array}{c}
n-1 \\
j
\end{array}\right)\left(\begin{array}{c}
\alpha j+\alpha-1 \\
k
\end{array}\right)\left(\begin{array}{l}
k \\
i
\end{array}\right)(-1)^{j+k} \frac{n \alpha \lambda^{k-i+2}}{(\lambda+1)^{k-i+1}} \\
\times\left[\frac{\Gamma(m+k-i+1)}{(\lambda k+\lambda)^{m+k-i+1}}+\frac{\Gamma(m+k-i+2)}{(\lambda k+\lambda)^{m+k-i+2}}\right]
\end{aligned}
$$

for $\alpha, \lambda>0$ and $0<\theta<1$. 


\section{A NEW LIFETIME DISTRIBUTION FOR SERIES SYSTEM}

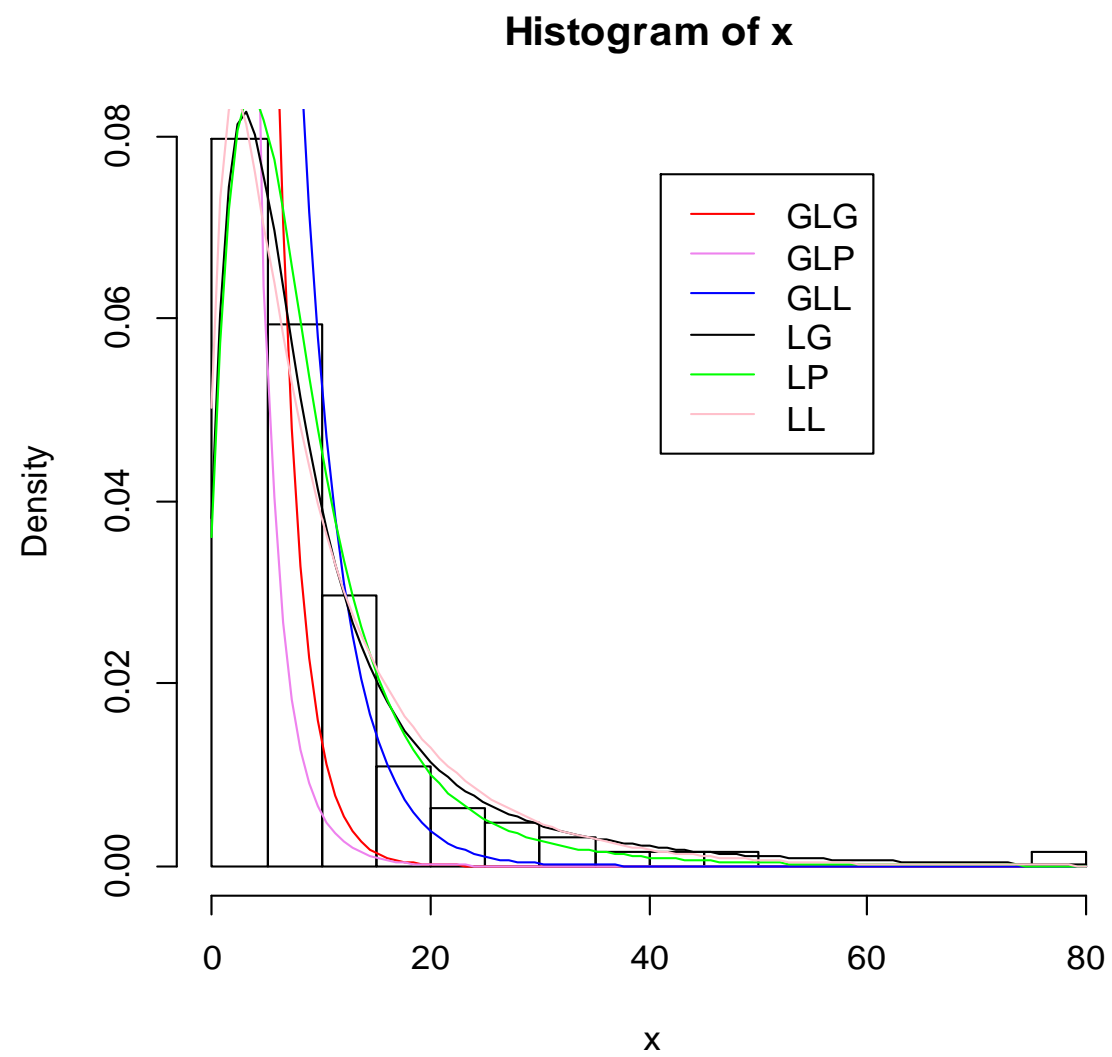

Figure 7. Fitting of GLP, GLG, GLL, LG, LP, and LL distributions to the cancer data

\section{Generalized Lindley Binomial Distribution}

The binomial distribution is a particular case of the PSD for $\mathrm{C}(\theta)=(\theta+1)^{m}+1$, with $m$ a positive integer, and a compound of generalized Lindley binomial (GLB) distribution follows from (2):

$$
\mathrm{F}(x)=1-\frac{\left(\left(1-\left[1-\left(1+\frac{\lambda x}{\lambda+1}\right) \mathrm{e}^{-\lambda x}\right]^{\alpha}\right) \theta+1\right)^{m}-1}{(\theta+1)^{m}-1}, \quad x>0
$$

The associated pdf, hazard rate, and survival functions are 


\section{RASHID ET AL}

$$
\begin{gathered}
\mathrm{f}(x)=\frac{m \alpha \lambda^{2} \theta \mathrm{e}^{-\lambda x}(1+x)}{(\lambda+1)\left((\theta+1)^{m}-1\right)}\left[1-\left(1+\frac{\lambda x}{\lambda+1}\right) \mathrm{e}^{-\lambda x}\right]^{\alpha-1} \\
\times\left(\left(1-\left[1-\left(1+\frac{\lambda x}{\lambda+1}\right) \mathrm{e}^{-\lambda x}\right]^{\alpha}\right) \theta+1\right)^{m-1} \\
\mathrm{~h}(x)=m \alpha \lambda^{2} \theta(1+x) \mathrm{e}^{-\lambda x}\left[1-\left(1+\frac{\lambda x}{\lambda+1}\right) \mathrm{e}^{-\lambda x}\right]^{\alpha-1} \\
\times\left(\left(1-\left[1-\left(1+\frac{\lambda x}{\lambda+1}\right) \mathrm{e}^{-\lambda x}\right]^{\alpha}\right) \theta+1\right)^{m-1} \\
\left.\times\left[(\lambda+1)\left(\left(1-\left[1-\left(1+\frac{\lambda x}{\lambda+1}\right) \mathrm{e}^{-\lambda x}\right]^{\alpha}\right) \theta+1\right)^{m}-1\right)\right]^{-1} \\
\mathrm{~S}(x)=\frac{\left(\left(1-\left[1-\left(1+\frac{\lambda x}{\lambda+1}\right) \mathrm{e}^{-\lambda x}\right]^{\alpha}\right) \theta+1\right)^{m}-1}{(\theta+1)^{m}-1}
\end{gathered}
$$

respectively, for $x, \lambda, \alpha>0$ and $0<\theta<\infty$. The expression for the $r^{\text {th }}$ moment of a random variable following generalized Lindley binomial distribution becomes, by taking $a_{n}=\left(\begin{array}{c}m \\ n\end{array}\right)$ and $\mathrm{C}(\theta)=(\theta+1)^{m}+1$ in (6),

$$
\begin{aligned}
\mathrm{E}\left(X^{r}\right)=\sum_{n=1}^{\infty} \sum_{j=0}^{n-1} \sum_{k=0}^{\infty} \sum_{i=0}^{k}\left(\begin{array}{l}
m \\
n
\end{array}\right) \theta^{n}\left(\begin{array}{c}
n-1 \\
j
\end{array}\right)\left(\begin{array}{c}
\alpha j+\alpha-1 \\
k
\end{array}\right)\left(\begin{array}{l}
k \\
i
\end{array}\right)(-1)^{j+k} \frac{n \alpha \lambda^{k-i+2}}{(\lambda+1)^{k-i+1}} \\
\times\left[\frac{\Gamma(m+k-i+1)}{(\lambda k+\lambda)^{m+k-i+1}}+\frac{\Gamma(m+k-i+2)}{(\lambda k+\lambda)^{m+k-i+2}}\right]
\end{aligned}
$$

for $\mathrm{n} \leq \mathrm{m}, \alpha, \lambda>0$, and $0<\theta<\infty$. The new family of LPS distributions due to Adil and Jan (2016) is obtained by taking $\alpha=1$ in (1). Sub-models GLP, GLG, GLL, and GLB are new compound distributions. The graphical representation of the density function and hazard rate function of all the sub-models of the proposed family have also been presented. 


\section{A NEW LIFETIME DISTRIBUTION FOR SERIES SYSTEM}

Table 2. Parameter estimates, log likelihood, and Akaike Information Criterion (AIC)

\begin{tabular}{rrrr} 
Model & Maximum Likelihood Estimates & Log-likelihood & AIC \\
\hline GLG & $\hat{\lambda}=0.06, \hat{\theta}=0.92, \hat{\alpha}=1.07$ & -409.31 & 824.62 \\
GLP & $\hat{\lambda}=0.08, \hat{\theta}=3.65, \hat{\alpha}=0.86$ & -410.42 & 826.84 \\
GLL & $\hat{\lambda}=0.11, \hat{\theta}=0.96, \hat{\alpha}=1.22$ & -411.20 & 828.41 \\
LG & $\hat{\lambda}=0.07, \hat{\theta}=0.88$ & -409.59 & 823.18 \\
LP & $\hat{\lambda}=0.11, \hat{\theta}=3.17$ & -411.38 & 826.76 \\
LL & $\hat{\lambda}=0.12, \hat{\theta}=0.90$ & -411.77 & 827.54 \\
\hline
\end{tabular}

\section{Application}

The applicability of the proposed model will be explored further. A comparison among its sub-classes will also be undertaken using a real life data set based on the remission times (in months) of a random sample of 128 bladder cancer patients as reported in Lee and Wang (2003). The parameter estimates, log likelihood, and Akaike Information Criterion (AIC) are shown in Table 2.

\section{Conclusion}

A new lifetime distribution was proposed for series setting that not only generalizes the Lindley power series family of distribution, but has a flexible density function; more importantly, its hazard function can take up different shapes such as bathtub, upside down bathtub, increasing, and decreasing shapes. The potential of the proposed family has been shown by fitting it to the real life data set, and it is clear from the statistical analysis that the proposed family offers a better fit.

\section{Acknowledgements}

We are very thankful to the Editors comments and the publishing committee for efficiently reading the paper that truly improved the standard of paper.

\section{References}

Adamidis, K., Dimitrakopoulou, T., \& Loukas, S. (2005). On a generalization of the exponential-geometric distribution. Statistics \& Probability

Letters, 73(3), 259-269. doi: 10.1016/j.spl.2005.03.013 


\section{RASHID ET AL}

Adamidis, K., \& Loukas, S. (1998). A lifetime distribution with decreasing failure rate. Statistics \& Probability Letters, 39(1), 35-42. doi: 10.1016/s01677152(98)00012-1

Adil, R., \& Jan, T. R. (2016). A new family of lifetime distributions with real life applications. International Journal of Mathematics and Statistics, 17(1), 23-38.

Barakat, H. M., \& Abdelkader, Y. H. (2004). Computing the moments of order statistics from nonidentical random variables. Statistical Methods and Applications, 13(1), 15-26. doi: 10.1007/s10260-003-0068-9

Chahkandi, M., \& Ganjali, M. (2009). On some lifetime distributions with decreasing failure rate. Computational Statistics \& Data Analysis, 53(12), 44334440. doi: 10.1016/j.csda.2009.06.016

Kus, C. (2007). A new lifetime distribution. Computational Statistics \& Data Analysis, 51(9), 4497-4509. doi: 10.1016/j.csda.2006.07.017

Lee, E. T., \& Wang, J. (2003). Statistical methods for survival data analysis (3rd ed.). NewYork, NY: Wiley. doi: 10.1002/0471458546

Morais, A. L., \& Barreto-Souza, W. (2011). A compound class of Weibull and power series distributions. Computational Statistics \& Data Analysis, 55(3), 1410-1425. doi: 10.1016/j.csda.2010.09.030

Nadarajah, S., Bakouch, S. H., \& Tahmasbi, R. (2011). A generalized Lindley distribution. Sankhya B, 73(2), 331-359. doi: 10.1007/s13571-011-0025-9

Tahmasbi, R., \& Rezaei, S. (2008). A two-parameter lifetime distribution with decreasing failure rate. Computational Statistics \& Data Analysis, 52(8), 3889-3901. doi: 10.1016/j.csda.2007.12.002

Zakerzadeh, H., \& Mahmoudi, E. (2012). A new two parameter lifetime distribution: Model and properties. Unpublished manuscript, Department of Statistics, Yazd University, Yazd, Iran. Retrieved from https://arxiv.org/abs/1204.4248 